\title{
3D Radon Transform for Shape Retrieval Using Bag-of-Visual-Features
}

\author{
Jinlin Ma and Ziping Ma \\ College of Computer Science and Engineering, North Minzu University, China
}

\begin{abstract}
In order to improve the accuracy and efficiency of extracting features for 3D models retrieval, a novel approach using $3 D$ radon transform and Bag-of-Visual-Features is proposed in this paper. Firstly the $3 D$ radon transform is employed to obtain a view image using the different features in different angels. Then a set of local descriptor vectors are extracted by the SURF algorithm from the local features of the view. The similarity distance between geometrical transformed models is evaluated by using K-means algorithm to verify the geometric invariance of the proposed method. The numerical experiments are conducted to evaluate the retrieval efficiency compared to other typical methods. The experimental results show that the change of parameters has small effect on the retrieval performance of the proposed method.
\end{abstract}

Keywords: $3 D$ radon transform, Bag-of-Visual-Features, 3D models retrieval, K-means algorithm.

Received March 6, 2018; accepted January 28, 2020

https://doi.org/10.34028/iajit/17/4/5

\section{Introduction}

In recent years, extensive research efforts have been dedicated to 3D model retrieval and recognition. There are mainly two categories for 3D model retrieval and recognition: model-based methods and view-based methods. Model-based methods attempt to exploit the structure of the covariance interference matrix of 3D model. The drawbacks of model-based methods are model-mismatch and computationally expensive model fitting. These inconveniences hinder the improvement of 3D retrieval efficiency and limit the practical applications of model-based methods. The view-based methods concentrate on how to capture view images to represent a complicated 3D model. A view image can well depict the shape of a 3D model from all viewing angles because the two view images captured from two similar 3D models from a certain viewing angle are similar. However, the drawback is that these methods do not exploit semantic knowledge and discard invisible information of an object. Thus it is hard to reach final conclusion that some view-based method always performs perfectly in terms with all kinds of measures. Hence, it remains a challenging issue to investigate new view-based methods and improve retrieval performance in 3D model retrieval.

In this paper, we propose a novel method of feature extraction based on 3D Radon transform and BOVW algorithm. The empirical evaluation shows that the performance of the features is more efficient than the compared methods.

The remainder of this paper is organized as follows. In section 2, we introduce the related works, and analysis the various methods. In section 3 , we briefly review the recent research works on BOVF and 3D Radon transform. The proposed method based on 3D Radon moment and BOVF is defined in section 3. In section 4, the comparative experiments of the proposed approach with other methods are conducted in terms with the retrieval efficiency of 3D model, the influence of the number of codebook and the volume of views models on the retrieval performance. Section 5 is the conclusion of the paper.

\section{Related Works}

Furthermore, among view-based methods, a number of existing methods have employed different approaches such as Fourier coefficients [18], the Scale Invariant Feature Transform (SIFT) algorithm [13] and the elevation descriptor [20] to capture depth buffers. However, as a shape descriptor of a 3D model, a depth buffer is partial, rough and noisy, which makes it difficult to effectively match such data against a complete 3D model representation. Moreover, these methods need to use a large number of depth buffers, which inevitably aggravates computational cost.

Among view-based methods, Hypergraph plays an important role in hypergraph-based 3D object modeling and has been applied in many applied fields. A hypergraph can be constructed by different visual features such as view clustering [10], the correlation among different surface boundary segments [26, 29], the relationship between images in the visual feature spaces [12, 23], the images and the features of vertices [27], 2D views of a 3D model [9] and so on. In order to reduce the risk from applying a single hypergraph, multiple hypergraphs can be constructed by varying 
the number of clusters to capture a higher order relationship of $3 \mathrm{D}$ objects.

In addition, as a kind of view-based method and a popular technique to speed up the matching of image local features, the Bag-of-Features has recently been introduced into feature-based 3D shape retrieval. The Bag-of-Features relying on the extraction of spin image signatures which are later grouped in clusters is used for both global comparison and partial matching. In contrast, the bag-of-words model treats image features as a sparse histogram over the vocabulary. In computer vision, a bag of visual words is a vector of occurrence counts of a vocabulary of local image features. A number of research works are associated with a bag of visual words $[8,10,11,14,17,19,24]$. Furuya and Ohbuchi [8] proposed a multi-view bag of visual features algorithm shape-based 3D model retrieval. The shape descriptors based on appearance perform better in rigid and articulated 3D shapes with lower computational time than other methods having similar retrieval performance. An advantage of the method is its ability to deal with various kinds of 3D models such as polygon soup and point set. Another advantage is that it is invariant to rotation, articulation and global deformation of 3D shapes. However, classification is not necessarily desirable for similarity based 3D shape retrieval. Later, based on the previous research work, Ohbuchi [17] exploit the left issues such as incorporation of multi-class semantic and scalability in searching through a database. The progressive works include employing bag-of-features algorithm to obtain incorporating semantic. They also apply supervised and semi-supervised algorithm to reduce the feature size, feature vector and exploit semantic. However, the demerit of this method is the lack of the scalability of learning algorithms for dimension reduction [11].

In view clustering [10], a novel framework for 3D object categorization was proposed. After a hierarchical 3D object segmentation, the object's sub-parts geometric features are extracted and clustered for a fixed number of 3D visual words by k-means algorithm. Then, the object is categorized by Support Vector Machine (SVM). In [19], a 3D object is represented as a set of panoramic views and a Bag-ofVisual-Words model using SIFT algorithm for 3D object retrieval. The experimental results show that it is superior to state-of-the-art methods on standard datasets.

Generally, using one single feature to describe a 3D object is often insufficient. So many algorithms are proposed using multiple feature fusion. Wang [25] proposed a feature fusion method based on hypergraph for 3D object retrieval. The hypergraph adopted Zernike moments feature and Dense Kernel LBP feature as a fusion view feature. Chen et al. [2] proposed a multimodal support vector machine to combine three modalities features which are Sift descriptor, outline fourier transform descriptor, and
Zernike Moments descriptor. The method considered both the independence of each modality and the interrelation among them. Xiao et al. [28] proposed a fast view-based 3D model retrieval framework, which included Unsupervised Multiple Feature Fusion algorithm (UMFF) and efficient Online Projection Learning algorithm (OPL). The UMFF was a compact feature representation from multiple visual features, and the OPL transfered the multiple visual features into low-dimensional feature. Zhao et al. [31] proposed a feature fusion method for view-based 3D object retrieval, which extracted 2D Zernike moments, 2D Fourier descriptor and 2D Krawtchouk moments for feature fusion to describe each view of a 3D object.

Inspired by Bag-of-Visual-Words algorithm (BOVF), our approach employs 3D Radon transform in BOVW algorithm to extract shape features. As one of fundamental and useful tools in many areas [3, 6, 16], the 3D Radon transform can reflect the geometric structure of 3D model. Daras et al. [5] used the Radon projection matrix to construct a series of characteristic functions to describe shape feature. The experimental results showed that the shape descriptors had a good retrieval efficiency. However, they failed to verify the rotation, translation and scaling invariance of the feature. Daras et al. [4] proposed a general 3D radon transform using the radial integral transform of 3D radon transform, spherical integral transformation and enhanced radial integral transform. As translation invariance by translational center, the fusion features can achieve a good retrieval performance. In addition, Zarpalas [30] used the mapping matrix of the 3D Radon transform to construct a number of functions, and generated feature descriptor for model retrieval. Mahmoud and Shaker [15] applied 3D Radon transform and neural network to establish a human ear authentication algorithm, achieving a more perfect experimental result.

\section{The Method}

\subsection{Overview}

In this section, we first present an overview of our method and then elaborate on the details of each step in the corresponding subsections.

The proposed method is mainly based on 3D radon transform, BOVF algorithm and SURF algorithm. And the algorithm procedure depicted in Figure 1 is implemented subsequently in five steps:

- Step 1. Pre-process 3D models: Normalize 3D models with respect to the PCA algorithm.

- Step 2. Capture views: Use the 3D Radon transform to extract the projection information of 3D model from different perspectives and to obtain a view image using different angels $\theta$ and $\varphi$. 
- Step 3. Extract local features: The local features and

- Images obtained by using SURF algorithm. Then the feature points are classified by clustering method.

- Step 4. Construct histograms: A codebook is obtained using SURF descriptor by K-means algorithm to cluster the training data. Word histograms are built to count the frequency of the view word in the codebook. The final word histograms are as local feature vectors. Then the frequency of visual vocabulary of the model is counted to represent the BOVF features.

- Step 5. Match shapes: The similarity distance between geometrical transformed models is evaluated using Kullback-Leibler divergence (KLD) to measure the similarity between two 3D models.

\subsection{Capture the Views}

After all the models are normalized by PCA, the 3D Radon transform of a function $f(x)$ can be defined in spherical coordinates as:

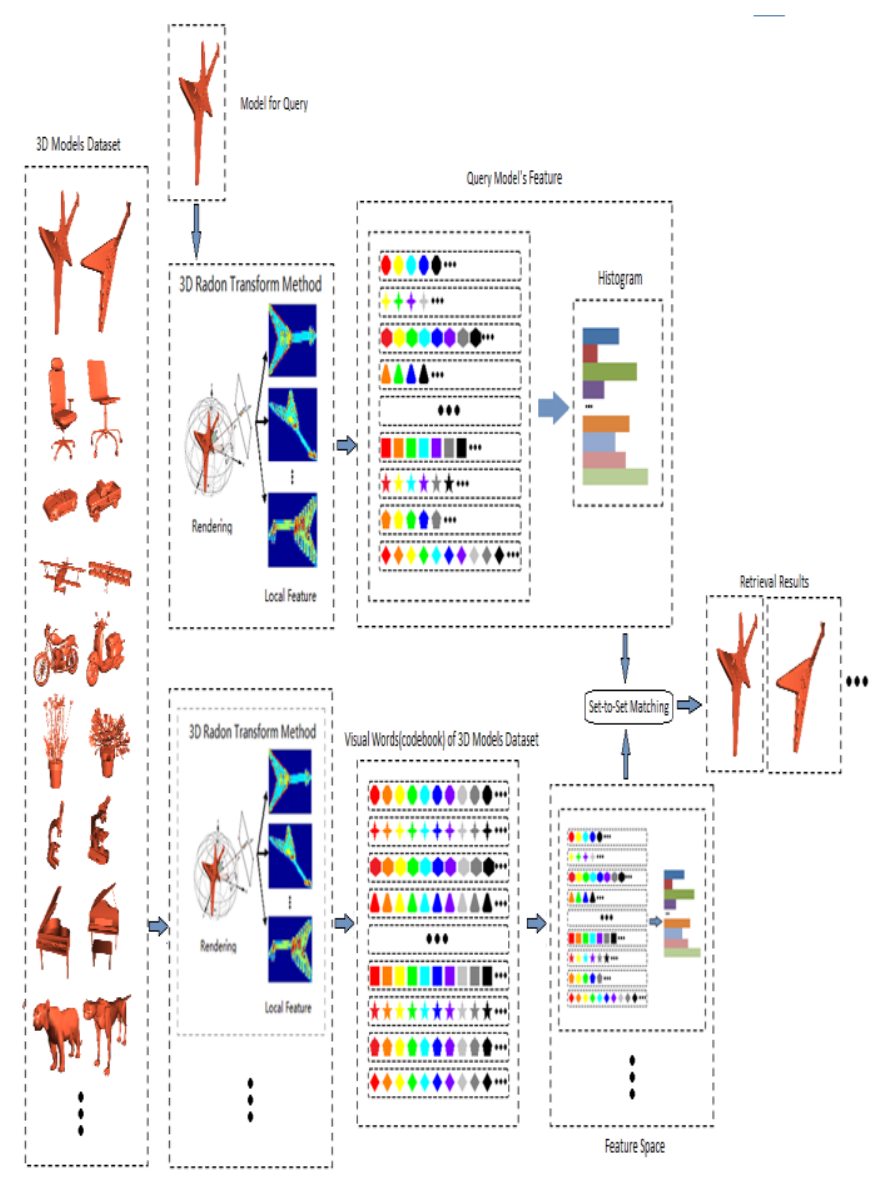

Figure 1. An illustration of our method.

$$
\begin{aligned}
R_{f}(\rho, \alpha, \beta) & =\iiint f(x) \delta(\rho-x \cos \beta \sin \alpha \\
& +y \sin \beta \cos \alpha+z \cos \alpha) d x d y d z
\end{aligned}
$$

Where $\rho$ is a real constant $R_{f}(\rho, \theta, \varphi)$ expresses the summation of values of $f(x)$ which lies on the plane by $(\rho, \theta, \varphi)$.

The views are captured from the projections of the function $f(x)$ on the plane of $(\rho, a, \beta)$ Figure 2 is an
SURF descriptors are extracted from these view illustrative diagram of the projections of the function $f(x)$ on the plane of $(\rho, a, \beta)$.

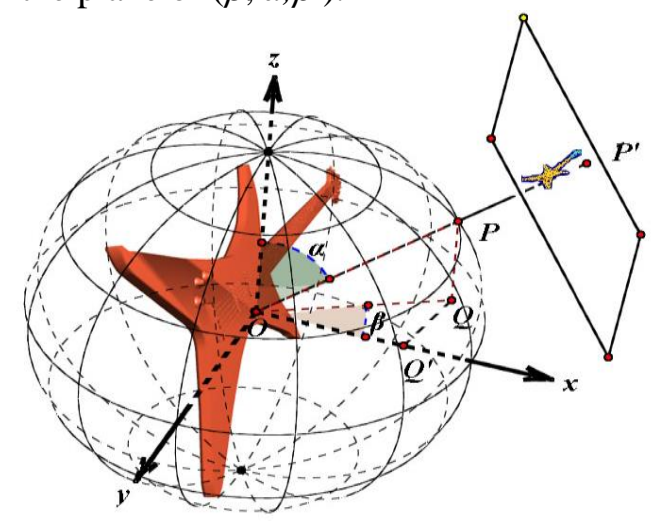

Figure 2. An illustration of the projections the function $f(x)$ on the plane of $(\rho \rho \alpha, \beta)$.

Figure 3 is a demonstration of capturing the multiviews. Theoretically, the parameter $\alpha$ and $\beta$ are among $(0,2 \pi]$. Let the parameter $\alpha$ and $\beta$ be $0^{\circ}$, $60^{\circ}, 120^{\circ}, 180^{\circ}, 240^{\circ}$ and $300^{\circ}$ respectively. Therefore, 36 views are obtained totally.

\subsection{Extract the Local Feature of the Views}

After capturing the multi-view images, SURF descriptors are obtained by detecting the interest point in the views. The first step consists of fixing a reproducible orientation based on information from a circular region around the interest point in the views. And secondly a square region aligned to the selected orientation is constructed, from which the SURF descriptor is extracted. The feature detector is based on multi-scale space theory and Hessian matrix.

\subsection{Bag of Visual Feature Construction}

In computer vision, the bag-of-words model can be applied to image classification by treating image features as words. In document classification, a bag of words is a sparse vector of occurrence counts of words; that is, a sparse histogram over the vocabulary. In computer vision, a bag of visual words is a vector of occurrence counts of a vocabulary of local image features.

In order to reduce time consuming of SURF descriptors, we apply vector quantizing on the SURF descriptors, so that the object can be described as a set of the visual word (Histograms). Specifically, a codebook is firstly obtained using SURF descriptor of visual images by K-means algorithm to cluster the training data. Then centers of the clusters are selected as the feature vectors of visual words in the codebook. And the number of the clusters is the size of codebook. 


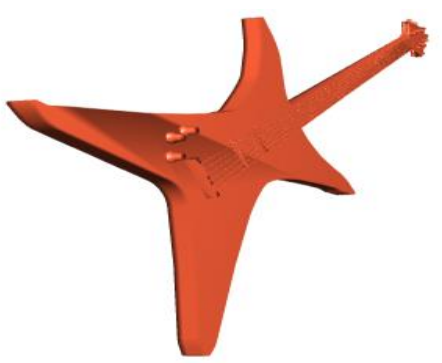

a) A 3D shape model.

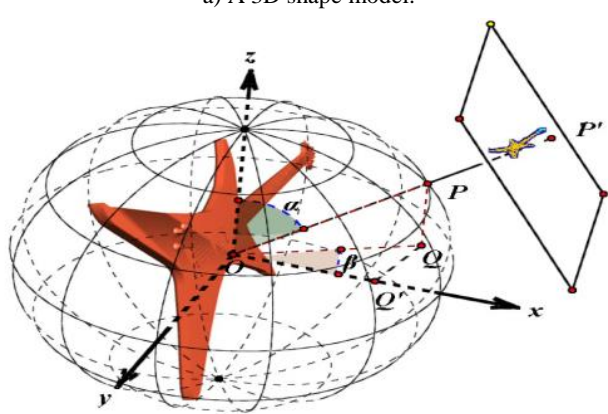

b) the views obtained by $3 \mathrm{D}$ radon transform on the plane $(\alpha, \beta)$.
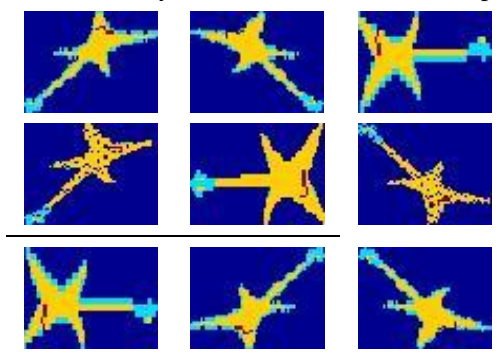

c) some of the captured views.

Figure 3. A demonstration of capturing the multi-views.

\subsection{Similarity Matching Between two Models}

Measure the similarity between two feature vectors by divergence KLD. It is not symmetric. KLD is a measure of the difference between two probability distributions of histogram vectors in visual dictionary.

$$
D(x, y)=\sum_{i=1}^{n}\left(y_{i}-x_{i}\right) \ln \frac{y_{i}}{x_{i}}
$$

Where $X=\left(X_{i}\right), y=\left(y_{i}\right)$ denote the feature vectors of $3 \mathrm{D}$ models, and $n$ is dimension of feature vectors.

\section{Experimental Evaluations}

In this section, we present our experimental results for 3D object retrieval. Firstly, we describe the test datasets. And then we present the experimental results and make comparisons with different typical methods for 3D object retrieval. The experimental results are discussed to study the influence of the number of codebook and the volume of views models on the retrieval performance for our method.
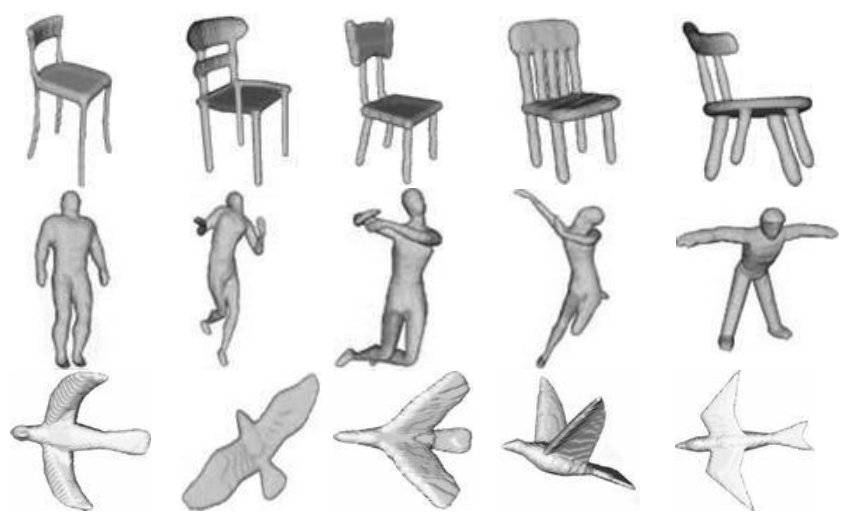

a) Example 3d models from the McGill datasets.
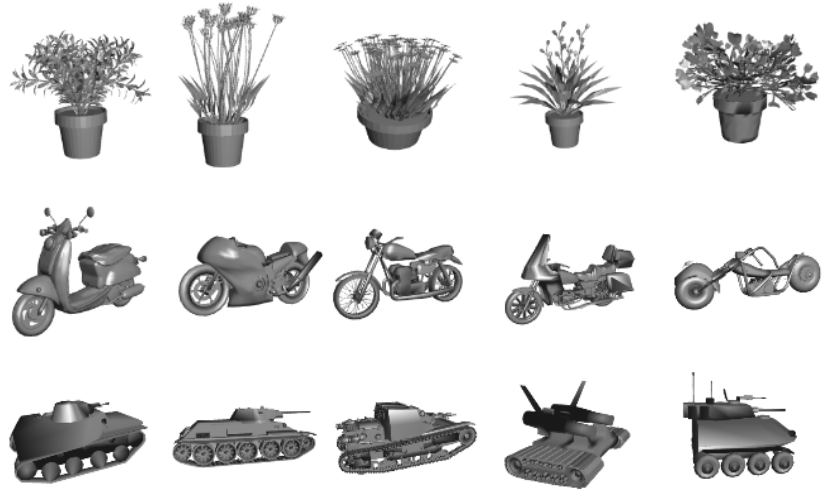

b) Example 3D models from the NSB datasets.
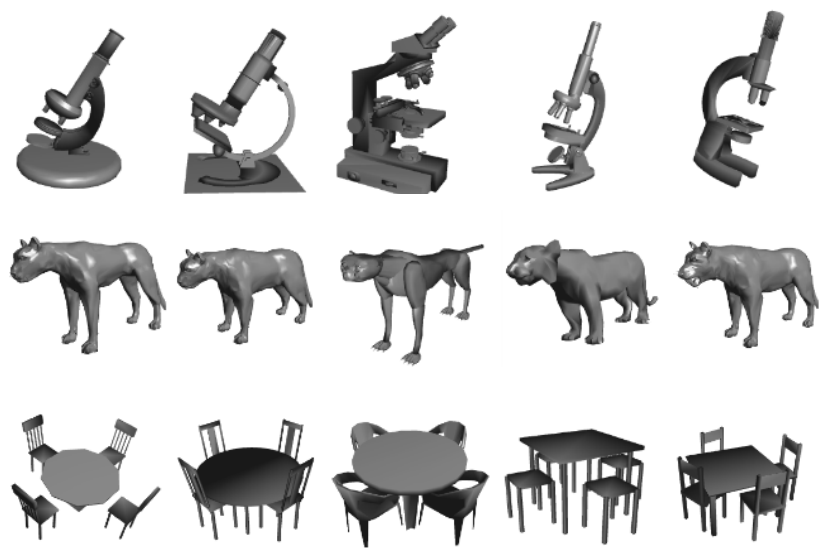

c) Example 3D models from the PSB datasets.
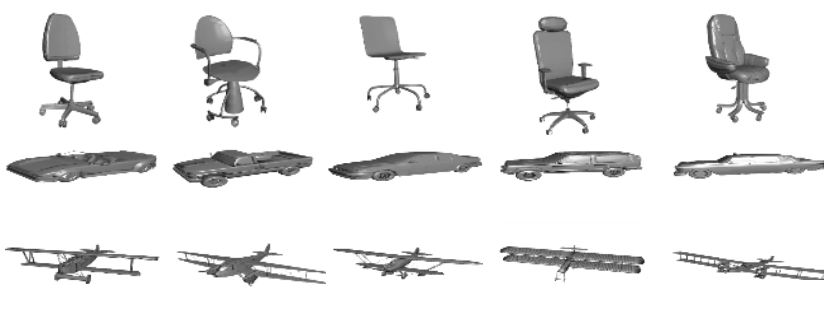

d) Example 3D models from the KDW datasets.

Figure 4. Example 3D models from the McGill, NSB, PSB and KDW datasets.

\subsection{Testing Datasets and Evaluation}

The performance of the proposed method is evaluated on four different databases: the McGill Shape benchmark [22], the NSB Shape benchmark [7], and the Princeton Shape Benchmark [21], and KDW datasets [1]. The first one contains 255 objects which are classified into 10 categories. The minimum 
number of objects per category is 20 , while the maximum number is 31 . The second one chosen from NSB contains 800 generic models which are classified into 40 categories. And each class contains 20 objects. The third one, formed from the PSB, consists of 907 models with 92 categories. And the minimum number of objects per category is 4, while the maximum number is 50 . The last one selected from KDW and SHREC'09 dataset [1] contains 360 models with 18 categories. The figure 4 presents some example models of the McGill, NSB, PSB and KDW datasets respectively.

In order to measure the efficiency of the methods, we take six kinds of evaluation measures which are based on the Precision-Recall curves (PR) curves and five quantitative measures including $\mathrm{NN}$ (the Nearest Neighbor), the First Tier (FT), the second Tier (ST), eMeasure (E-M) and the Discounted Cumulative Gain (DCG)[27].

\subsection{Comparison with the State-of-the-Art}

In the first experiment, we verify the retrieval performance in four databases mentioned above by comparing our method with ED, CM-BOF, SH, 3D Zernike and Polar method. The findings suggest that the default parameters of our proposed method are selected as follows: the volume size of test 3-D model is $90 * 90 * 90$; the number of view images is 25 and the size of codebook is 1000 in this experiment.

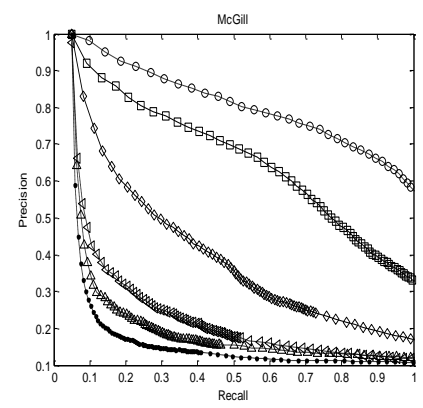

a) McGill.

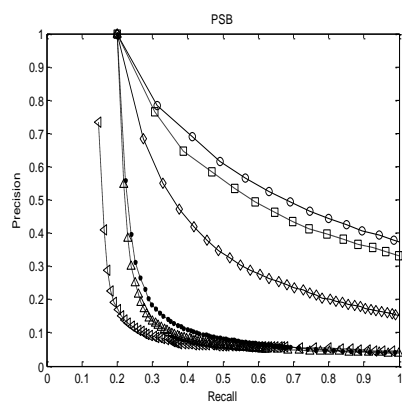

c) PSB.

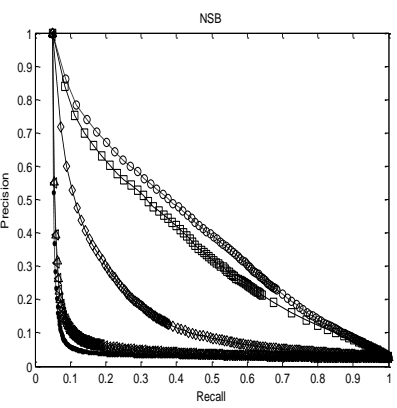

b) NSB

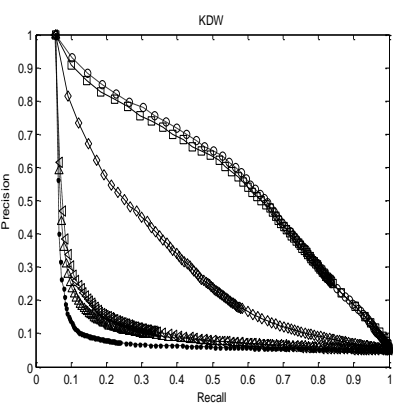

d) KDW.

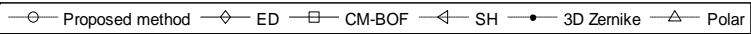

Figure 5. The precision-recall curves of different methods under various databases.

Figure 5 shows the PR curves on four test databases respectively. It can be observed that the proposed method always outperforms other compared methods in four databases, especially in McGill database where the contrast efficiency is the most significant. Among these compared methods, CM-BOF and ED take the second place and the third place respectively in terms with retrieval efficiency. Moreover, the proposed method is superior to CM-BOF methods so as to multi-view image based methods.

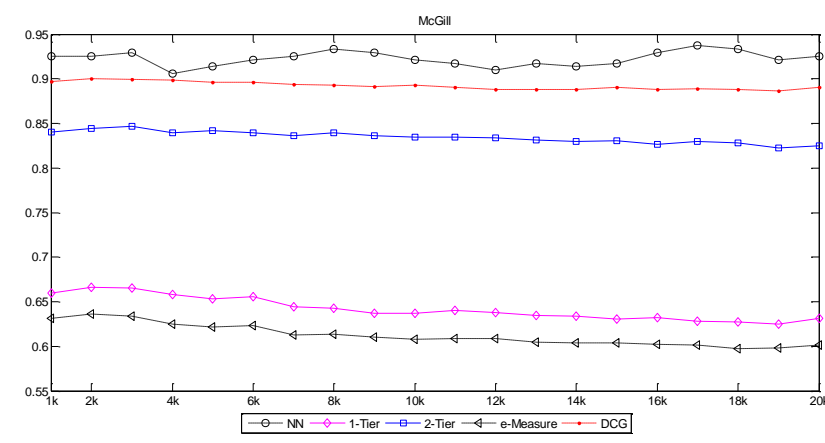

a) McGill.

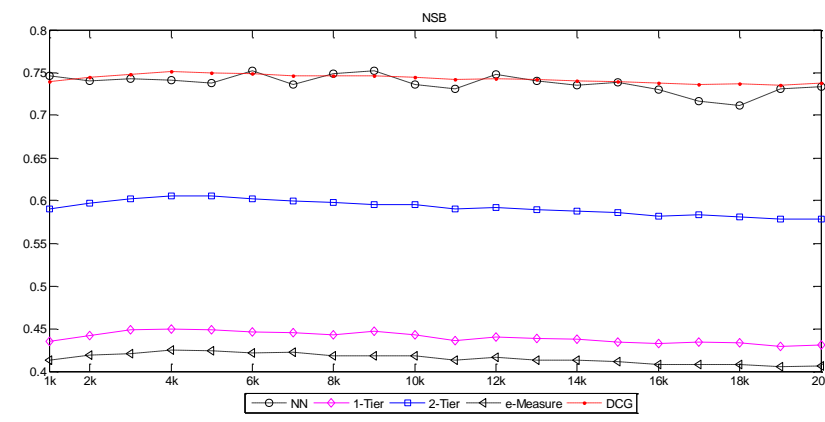

b) NSB.

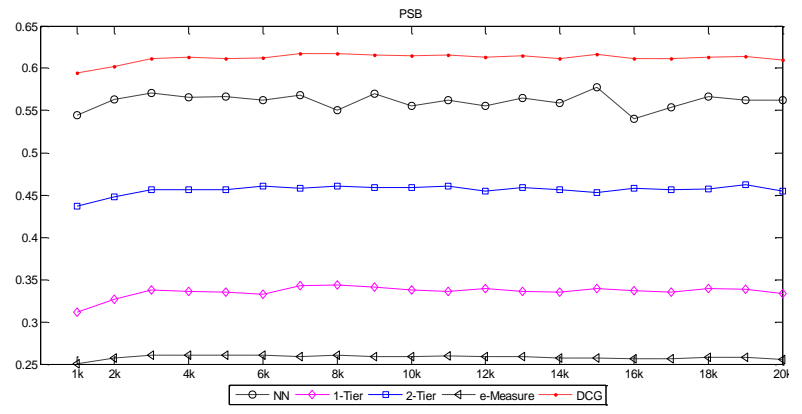

c) PSB.

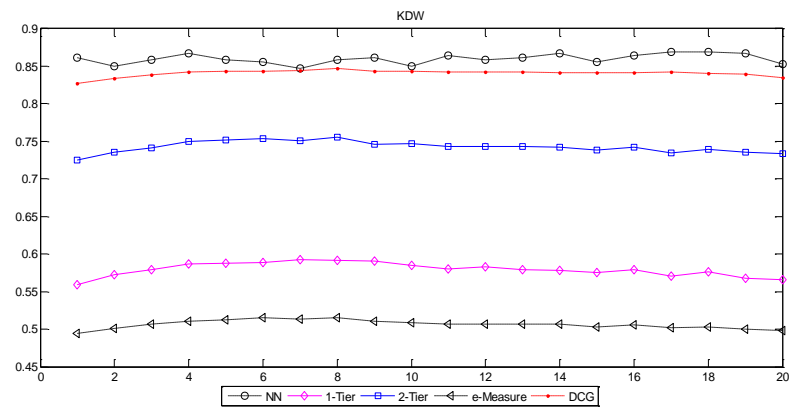

d) KDW.

Figure 6. The retrieval performances of different codebook size under various databases.

\subsection{Influence of the Codebook Size}

In the second experiment, we analyze the influence of the number of codebook on the retrieval performance of the proposed method. We conduct the experiment 
on four databases mentioned above in terms of precision of recall curve. Five quantitative measures include Nearest Neighbor (NN), First tier (1-Tier), Second Tier (2-Tier), Discounted cumulative Gain (DCG) and E-Measure. When the number of codebook varies from 1000 to 20000, the experimental results are shown in Figure 6. From the figure it can be observed that the curves of 1-Tier, 2-Tier, E-Measure and DCG almost keep level, which indicates that the retrieval performance of our method remains stable no matter what the number of codebook is. However, the shock is small, i.e. the mean of the shock through the amplitude of the curve of $\mathrm{NN}$ is small, which indicates that the retrieval performance of our method is slightly affected by codebook size. Furthermore, the values of $\mathrm{NN}$ and DCG are between 0.52 and 0.95 in four databases, especially in McGill and KDW databases where they are above 0.85 and 0.82 respectively. It demonstrates that the proposed method has effective retrieval performance to some extent.

Figure 7 depicts the influence of the number of codebook on the retrieval performance of our proposed in terms with P-R curves in four databases respectively. It can be observed that the corresponding P-R curves are same or nearly similar except that the P-R trend curves are slightly different in McGill database, which indicates that the retrieval performance remains stable and efficient no matter how large or small the number of codebook is. On the other hand, it also can be observed that the proposed method gets the best retrieval performance when the codebook size is 5000 in four databases.

However, the proposed method obtains the worst retrieval performance when the codebook size is 1000 and 20000 for McGill database and NSB database, as well as for PSB database and KDW database respectively. However, as shown in Table 1, the standard deviations of four quantitative measure values in test databases respectively are among [0.0023,0.0128], which demonstrates that the number of codebook size has less impact on the retrieval performance of the proposed method. It can be seen from Table 1 that the retrieval effect of McGill and KDW is good, and the values of NN, 1-tier, 2-tier, EMEASURE and DCG are higher than those of NSB and $\mathrm{PSB}$, which is also verified by the P-R curve in Figure 7.

In conclusion, the retrieval performance of our method has strong robustness to codebook size from the
Figures 6 and 7. Therefore, in practice it is proper that the number of codebook size is set to 5000 because it can obtain better retrieval efficiency of the algorithm while reducing the storage space.
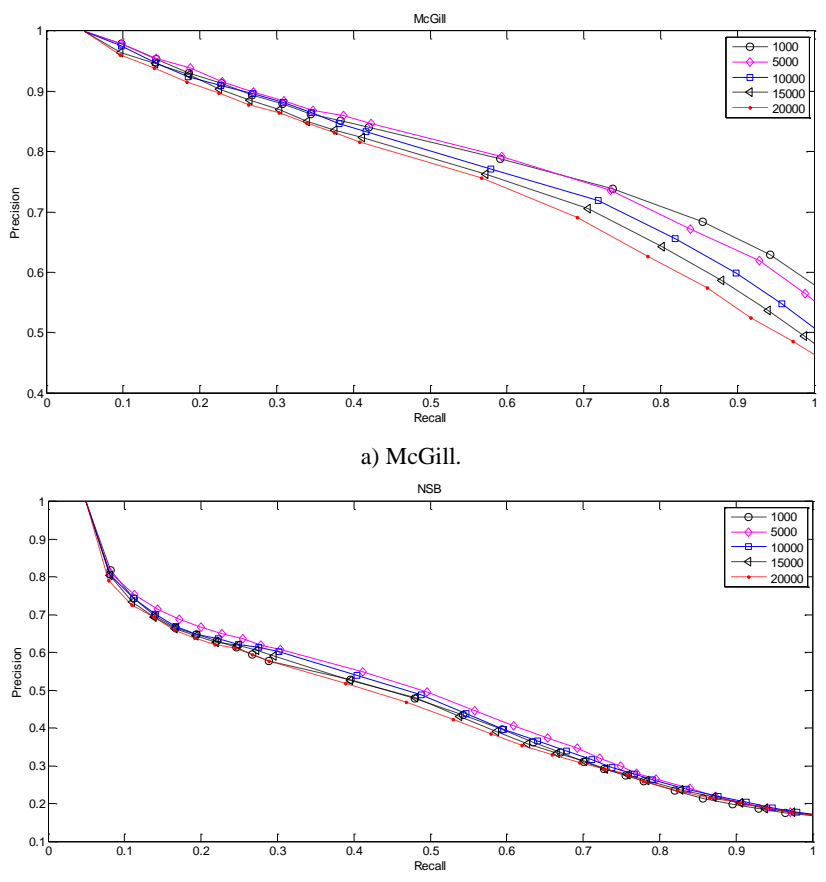

b) NSB.

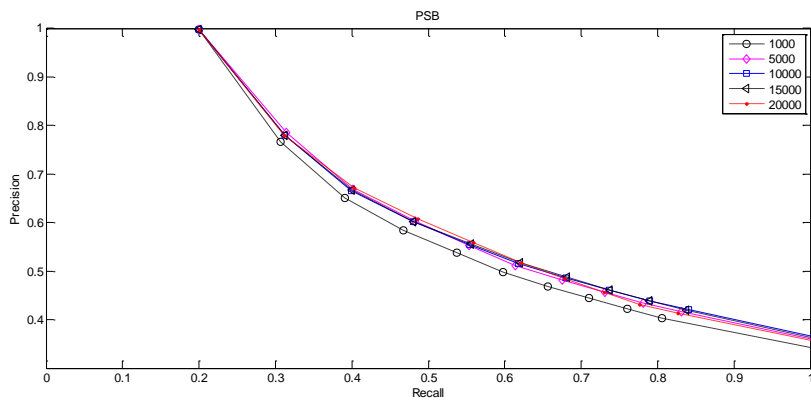

c) PSB

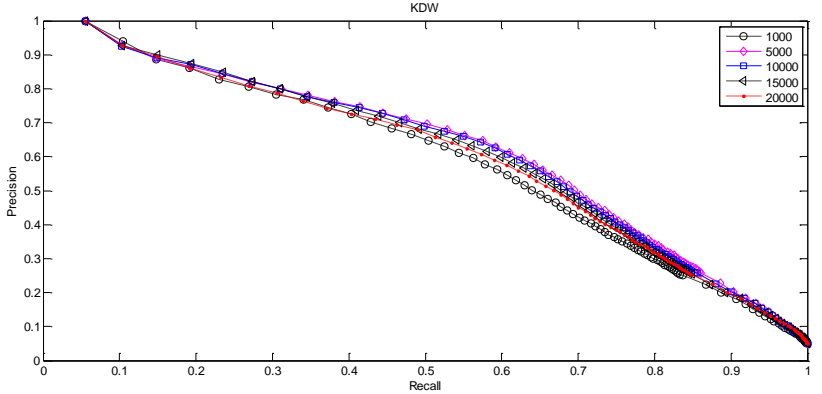

(d) KDW.

Figure 7. The precision-recall curves of the proposed method with different codebook size in test databases.

Table 1. The standard deviation of NN, 1-Tier, 2-Tier, E-Measure and DCG in test databases.

\begin{tabular}{|c|c|c|c|c|c|c|c|c|c|c|}
\hline \multirow[b]{2}{*}{$\begin{array}{l}\text { standard } \\
\text { deviation }\end{array}$} & \multicolumn{2}{|c|}{$\mathbf{N N}$} & \multicolumn{2}{|c|}{ 1-Tier } & \multicolumn{2}{|c|}{ 2-Tier } & \multicolumn{2}{|c|}{ E-Measure } & \multicolumn{2}{|c|}{ DCG } \\
\hline & mean & $\begin{array}{l}\text { standard } \\
\text { deviation }\end{array}$ & mean & $\begin{array}{l}\text { standard } \\
\text { deviation }\end{array}$ & mean & $\begin{array}{l}\text { standard } \\
\text { deviation }\end{array}$ & mean & $\begin{array}{l}\text { standard } \\
\text { deviation }\end{array}$ & mean & $\begin{array}{l}\text { standard } \\
\text { deviation }\end{array}$ \\
\hline McGill & 0.9227 & 0.0081 & 0.6420 & 0.0128 & 0.8345 & 0.0065 & 0.6124 & 0.0118 & 0.8922 & 0.0043 \\
\hline NSB & 0.7374 & 0.0102 & 0.4399 & 0.0063 & 0.5920 & 0.0085 & 0.4151 & 0.0060 & 0.7426 & 0.0047 \\
\hline PSB & 0.5611 & 0.0088 & 0.3357 & 0.0067 & 0.4561 & 0.0055 & 0.2581 & 0.0023 & 0.6121 & 0.0051 \\
\hline KDW & 0.8597 & 0.0064 & 0.5794 & 0.0089 & 0.7426 & 0.0074 & 0.5063 & 0.0056 & 0.8407 & 0.0043 \\
\hline
\end{tabular}




\subsection{Influence of the Number of View Images}

Furthermore, the influence of the number of view images on the retrieval performance of the proposed method has been investigated. We conduct the experiment on four databases in terms with the five quantitative measures mentioned above when codebook size is 1000 ; the size of volume is $100 * 100 * 100$ and the number of views image varies from $3 * 3$ to $9 * 9$. According to figure 8, the experimental results illustrate that the five quantitative measures almost remain unchanged when the number of view images varies from $3 * 3$ to $9 * 9$ except that it slightly decreases in the case of $4 * 4$, which reveals that the number of view images has little impact on the retrieval performance of the proposed method.

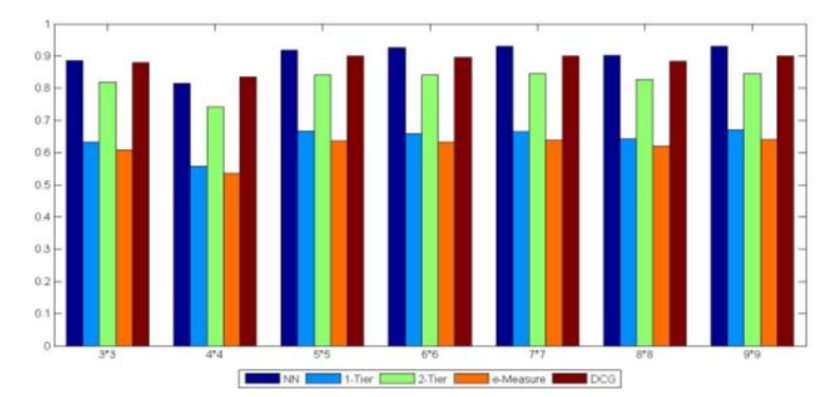

Figure 8. The bar graph of performance comparison of the proposed method with different number of views on the McGill.

Moreover, when the number of view images is $5 * 5$, the values of the five quantitative measures are respectively $98.73 \%, 99.52 \%, 99.70 \%, 99.64 \%$ and $99.98 \%$ of those when the number of view images is $9 * 9$. Meanwhile, the proportion of the numbers of view images in these two cases is only $30.86 \%$, so it is suitable to set the number of view image to $5 * 5$ in practice since it contributes to better retrieval performance as well as less computer storage space.

\subsection{Influence of Volume Size of 3D Model}

Finally we also employ the five quantitate measures to evaluate the retrieval efficiency of the proposed method with different volume size of 3D-model. The experiment is carried out in the McGill database in terms with $3 \mathrm{D}$-model volume varying from $20 * 20 * 20$ to $160 * 160 * 160$, codebook size 1000 and view size $6 * 6$. The compared values of five quantitative measures are demonstrated in the Figure 9:

- The values of five quantitative measures increase slightly with volume increasing from $20 * 20 * 20$ to $60 * 60 * 60$ and the increment speed is gradually and slightly slow when volume size is from $70 * 70 * 70$ to $140 * 140 * 140$.

- The values of five quantitative measures in case of $130 * 130 * 130$ are almost the same as those in case of $140 * 140 * 140$ and $150 * 150 * 150$. However, when volume size is $70 * 70 * 70$, the values of the five quantitative measures are $93.03 \% 90.57 \%, 95.33 \%$
, $92.28 \%$ and $96.91 \%$ respectively of those in case of the highest values, i.e., $160 * 160 * 160$. Meanwhile, the proportion of volume sizes in two cases is $15.61 \%, 12.5 \%, 12.5 \%, 12.5 \%$ and $10.16 \%$ respectively instead. Hence, it is suitable to choose the volume size $70 * 70 * 70$ in practice in consideration of the retrieval efficiency and restoration space.

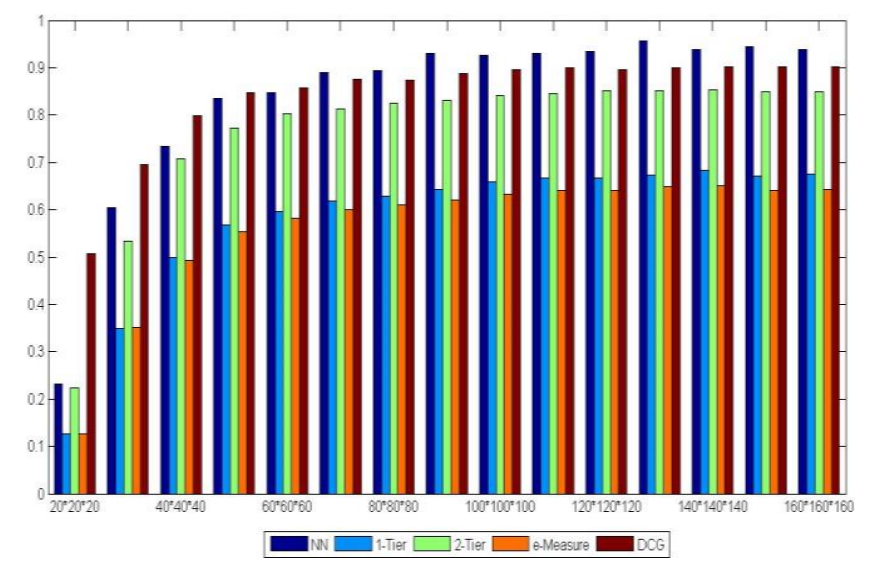

Figure 9. The influence of five quantitative measures in terms with different volume size on the McGill database.

\section{Conclusions}

In this paper, we presented a novel method to extract 3D shape descriptors based on 3D Radon transform and BOVF method for 3D models retrieval. The numerical experiments are conducted to evaluate the retrieval efficiency compared to other typical methods. The retrieval experimental results reveal that the proposed method consistently performs better or are comparable to the best results. More experiments are conducted and discussed to verify the influence of the different parameters. The parameters change has small effect on the retrieval performance of the proposed method.

Moreover, the advantages of the proposed method can exert high efficiency for content-based image retrieval and can be expanded to other application fields in future.

\section{Acknowledgements}

This work is supported by the National Natural Science Foundation of China (Grant No. 61462002), Innovation team of image and intelligent information processing and Computer vision and virtual reality of north minzu university, research project of north Minzu University (2018xyzjk02), education and teaching reform project of north minzu university (no.2018zhjy01). The authors would like to thank the anonymous referees for their valuable comments and suggestions. 


\section{References}

[1] Akgul C., Axenopoulos C., Bustos B., Chaouch M., Daras P., Dutagaci H., Godill A., Furuya T., Godil A., Kreft S., Lian Z., and Napoleon T., "SHREC 2009-Generic Shape Retrieval Contest," in Proceedings of Eurographics Workshop on 3D Object Retrieval, Munich, pp. 61-68, 2009.

[2] Chen F., Ji R., and Cao L., "Multimodal Learning for View-Based 3D Object Classification," Neurocomputing, vol. 195, pp. 23-29, 2016.

[3] D'Acunto M., Benassi A., Moroni D., and Salvetti O., "3D Image Reconstruction Using Radon Transform," Signal, Image and Video Processing Journal, vol. 10, no. 1, pp. 1-8, 2016.

[4] Daras P., Zarpalas D., Tzovaras D., and Strintzis M., "Efficient 3-D Model Search and Retrieval Using Generalized 3-D Radon Transforms," IEEE Transactions on Multimedia, vol. 8, no. 1, pp. 101-114, 2006.

[5] Daras P., Zarpalas D., Tzovaras D., and Strintzis M., "Shape Matching Using The 3D Radon Transform," in Proceedings of the $2^{\text {nd }}$ International Symposium on 3D Data Processing, Visualization, and Transmission, Thessaloniki, pp. 953-960, 2004.

[6] Desbat L. and Perrier V., "on Locality of Radon to Riesz Transform," Signal Processing, vol. 120, pp. 13-25, 2016.

[7] Fang R., Godil A., Li X., and Wagan A., "A New Shape Benchmark for 3D Object Retrieval," in Proceedings of the $4^{\text {th }}$ International Symposium on Advances in Visual Computing, Las Vegas, pp. 381-392, 2008.

[8] Furuya T. and Ohbuchi R., "Dense Sampling and Fast Encoding for 3D Model Retrieval Using Bag-of-Visual Features," in Proceedings of the ACM International Conference on Image and Video Retrieval, New York, pp. 1-8, 2009.

[9] Gao Y. and Dai Q., "Efficient View-Based 3-D Object Retrieval via Hypergraph Learning," Tsinghua Science and Technology, vol. 19, no. 3, pp. 250-256, 2014.

[10] Gao Y., Wang M., Tao D., Ji R., and Dai Q., “3-D Object Retrieval and Recognition with Hypergraph Analysis," IEEE Transactions on Image Processing, vol. 21, no. 9, pp. 4290-4303, 2012.

[11] Lian Z., Godil A., and Sun X., "Visual Similarity Based 3D Shape Retrieval Using Bag-ofFeatures," in Proceedings of Shape Modeling International Conference, Washington, pp. 25-36, 2010.

[12] Liu Y., Wang X., Wang H., Zha H., and Qin H., "Learning Robust Similarity Measures for 3D Partial Shape Retrieval," International Journal of Computer Vision, vol. 89, no. 2, pp. 408-431, 2010.
[13] Lowe D., "Distinctive Image Features from Scale-Invariant Keypoint," International Journal of Computer Vision, vol. 60, no. 2, pp. 91-110, 2004.

[14] Ma J., Kang B., Ma Z., and Lu K., "A Method of Protein Model Classification and Retrieval Using Bag-of-Visual-Features," Computational and Mathematical Methods in Medicine, 2014

[15] Mahmoud W. and Shaker M., "3D Ear Print Authentication Using 3D Radon Transform," Information and Communication Technologies, pp. 1052-1056, 2006.

[16] Miaoa Q., Liua J., and Lib W., "Three Novel Invariant Moments Based on Radon and Polar Harmonic Transforms," Optics Communications, vol. 285, no. 6, pp. 1044-1048, 2012.

[17] Ohbuchi R., "Squeezing Bag-of-Features for Scalable and Semantic 3D Models Retrieval," in Proceedings of the $8^{\text {th }}$ International Workshope on Content-Based Multimedia Indexing, 2010.

[18] Passalis G., Theoharis T., and Kakadiaris I., "PTK: A Novel Depth Buffer-Based Shape Descriptor for Three-Dimensional Object Retrieval," The Visual Computer, vol. 23, no. 1, pp. 5-14, 2007.

[19] Sfikas K., Theoharis T., and Pratikakis I., "3D Object Retrieval via Range Image Queries in a Bag-of-Visual-Words Context," The Visual Computer, vol. 29, no. 12, pp. 1351-1361, 2013.

[20] Shih J., Lee C., and Wang J., "A New 3D Model Retrieval Approach Based on the Evevation Descriptor," Pattern Recognition, vol. 40, no. 1, pp. 283-295, 2007.

[21] Shilane P, Min P., and Kazhdan M., "The Princeton Shape Beachmark," in Proceedings of the Shape Modeling International, pp. 167-178, 2004.

[22] Siddiqi K., Zhang J., and Macrini D., "Retrieving articulated 3-d models using medial surfaces," Machine Vision and Applications, vol. 19, pp. 261-275, 2008.

[23] Silkan H., Ouatik S., and Lachkar A., "Extreme Curvature Scale Space for Efficient Shape Similarity Retrieval," The International Arab Journal of Information Technology, vol. 13, no. 6A, pp. 203-207, 2016

[24] Toldo R., Castellani U., and Fusiello A., "A Bag of Words Approach for 3D Object Categorization," in Proceedings of the 4th International Conference on Computer, Vision/ Computer Graphics Collaboration Techniques, pp. 116-127, 2009.

[25] Wang F., Peng J., and Li Y., "Hypergraph Based Feature Fusion for 3-D Object Retrieval," Neurocomputing, no. 151, pp. 12-619, 2015.

[26] Wong A. K. C., Lu S. W., and Rioux M., "Recognition and Shape Synthesis of 3-D Objects Based on Attributed Hypergraphs," 
IEEE Transactions on Pattern Analysis and Machine Intelligence, vol. 11, no. 3, pp. 279-290, 1989.

[27] Xia S., and Hancock E. R., "Learning Large Scale Class Specific Hyper Graphs for Object Recognition," in Proceedings of the $5^{\text {th }}$ International Conference on Image and Graphics, Perth Australia pp. 366-371, 2009.

[28] Xiao J., Fengn Y., and Ji M., "Fast View-Based 3D Model Retrieval Via Unsupervised Multiple Feature Fusion and Online Projection Learning," Signal Processing, no. 120, pp. 702-713, 2016.

[29] Yuvaraj J., and Hariharan S., "Content-Based Image Retrieval Based on Integrating Region Segmentation and Colour Histogram," The International Arab Journal of Information Technology, Vol. 13, No. 1A, pp. 203-207, 2016

[30] Zarpalas D., Daras P., and Tzovaras D., "3D Model Search and Retrieval Based on the 3D Radon Transform," in Proceedings of IEEE International Conference on Communications, pp. 1375-1379, 2004.

[31] Zhao S., Yao H., and Zhang Y., "View-based 3D object retrieval via multi-modal graph learning," Signal Processing, no. 112, pp. 110-118, 2015.

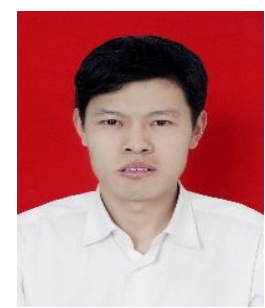

Jinlin Ma $\mathrm{He}$ received the B.S. degree in Computer Institute from North University for Nationalities, Yinchuan, China in 1999 and received the M.S. degree in Institute of Mathematics \& Computer from Ningxia University, Yinchuan, China in 2009. He received his Ph.D. degree at Northwest University, Xi'an, China in 2015. His research interests is in image processing and $3 \mathrm{D}$ models retrieval.

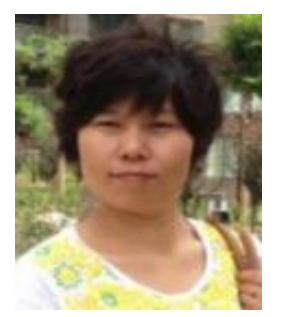

Ziping Ma She received the B.S. degree in Computer Institute from North University for Nationalities, Yinchuan, China in 2003 and received the M.S. degree in Institute of Mathematics \& Computer from Ningxia University, Yinchuan, China in 2006. She received her Ph.D. degree at Northwest University, Xi'an, China in 2013. Her research interests is in image processing and 3D models retrieval. 